
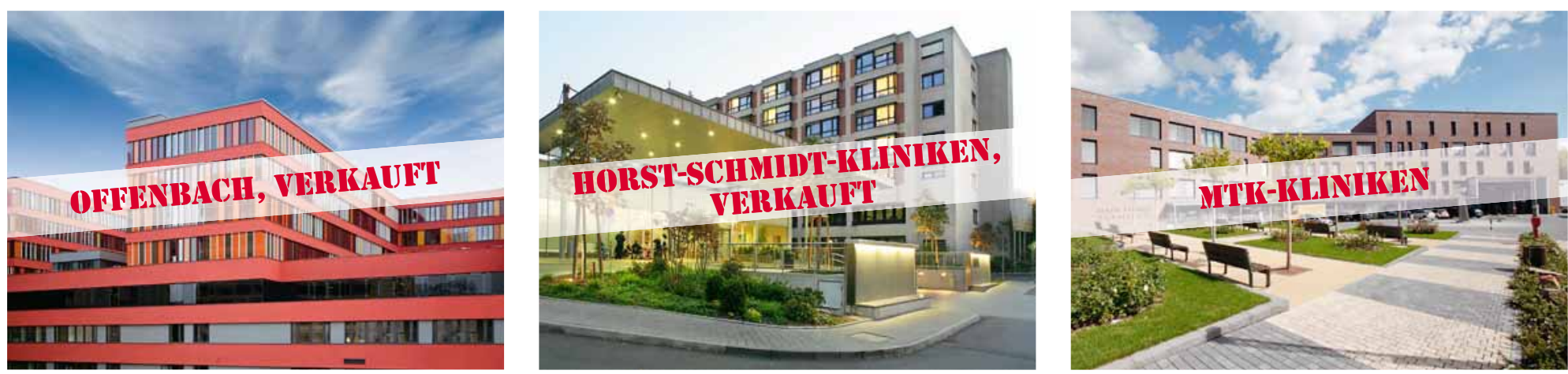

\title{
Ausweg verzweifelt gesucht
}

\author{
Hessens kommunale Kliniken sind großteils defizitär. Ein Gutachten des Landesrech- \\ nungshofs zeigt nun, wie kurzsichtig die Kliniken teilweise investiert haben. Derweil \\ bastelt das hessische Gesundheitsministerium weiter am Konstrukt einer hessischen \\ Klinik-Stiftung. Die Erfolgsaussicht ist jedoch spärlich.
}

$\mathbf{W}$ ieso baut ein Haus eine $\mathrm{Ge}$ burtshilfe, wenn die Nachbarkommune bereits eine vorhält? Weil Landräte und Stadtkämmerer nicht über den Tellerrand schauen wollen, lautet die einfache Antwort. In Hessen ist das nicht anders als vielerorts in Deutschland: Statt zu kooperieren, schlagen sich viele kommunale Kliniken als Einzelkämpfer und Konkurrenten durch. Fast alle Landesgesundheitsminister erzürnt dieser Umstand, doch in Hessen sind die Folgen besonders deutlich. Schon jetzt ist über ein Viertel der Klinikbetten in privater Hand, deutlich mehr als im Bundesdurchschnitt. Zuletzt traf es zwei Maximalversorger aus dem Rhein-Main-Gebiet: Wiesbaden und Offenbach gingen an Rhön und Sana. Viel verdient haben die Kommunen an diesen Veräußerungen nicht. Es waren Notverkäufe abgewirtschafteter Häuser - und die Lage im Rhein-Main-Gebiet, in dem die Krankenhausdichte besonders hoch ist, bleibt dramatisch. Mehrere öffentliche Maximalversorger graben sich in einem ruinösen Kampf um Patienten gegenseitig das Wasser ab.

\section{Ohne Abstimmung investiert}

Der Landesrechnungshof hat diesen planungspolitischen Irrsinn jetzt in einem Bericht veranschaulicht. Die Behörde hat sieben hessische Maximalversorger detailliert prüfen lassen. Sechs der Häuser machten 2011 Miese, summiert schrieben alle sieben 2011 einen Verlust von rund 79 Millionen Euro. Die Behörde wird in ihrer Kritik an den Kliniken und ihren Trägern sehr deutlich: Für die bauliche Infrastruktur und die Ausstattung der sieben Häuser wurden zwischen 2001 und 2011 rund 1,1 Milliarden Euro investiert. Allein 470 Millionen Euro davon wurden für die Kliniken des Main-TaunusKreises, des Hochtaunuskreises und in Frankfurt-Höchst genehmigt. „Diese drei Krankenhäuser liegen lediglich bis zu 25 Kilometer voneinander entfernt. Die Krankenhäuser hatten zu Beginn der Prüfung Mitte 2012 untereinander keine Abstimmungen ihrer baulichen Maßnahmen getroffen“, moniert der Rechnungshof. Er prangert fragwürdige Baumaßnahmen an, wie etwa das Trauma-Zentrum der Kliniken des Main-Taunus-Kreises (MTK) in Bad Soden mit Dachlandeplatz für Hubschrauber. In unmittelbarer „Flugnachbarschaft" befinden sich die Landeplätze der Uniklinik und der Berufsgenossenschaftlichen Unfallklinik in Frankfurt. Der Rechnungshof kritisiert auch einen Teilneubau für Konservative Medizin des Klinikums Darmstadt. „Die Kosten von 59 Millionen Euro dieser eigentlich wenig komplexen Baumaßnahme lagen - verglichen mit den Investitionen bei den anderen Krankenhäusern - im oberen Bereich. Trotzdem ist es nicht gelungen, mit dem Haus eine zukunftsfähige, flexibel nutzbare Infrastruktur zu schaffen.“

Ohne gemeinsame Strategie, so das Fazit des Rechnungshofs, werden Baumillionen unnötig verprasst. Am Ende müssen die klammen Kommunen die 


\section{„Kommunale müssen sich abstimmen“}

Der ehemalige Chef der Uniklinik Greifswald, Claus Bartels, ist heute Geschäftsführer von MedAdvisors. Die Beratungsagentur hat mit drei Partnern im Auftrag des hessischen Landesrechnungshofs sieben kommunale Kliniken Hessens unter die Lupe genommen.

Interview mit Claus Bartels, Geschäftsführer MedAdvisors

Welche neuen Erkenntnisse hat Ihre Studie gebracht?

Wir haben die Schwerpunkte der sieben Krankenhäuser - unabhängig von den Fachabteilungen - anhand von erwirtschafteten Case-Mix-Punkten in den einzelnen Hauptdiagnosegruppen (MDC) analysiert. So können wir Aussagen über die Leistungsstärke einzelner Krankenhäuser bei bestimmten Behandlungen treffen und sie mit den Zahlen umliegender Häuser ins Verhältnis setzen. Auf dieser Grundlage lässt sich relativ einfach unter- suchen, ob Fehlanreize bestehen und wie sie sich auswirken. Braucht dieses oder jenes Haus eine Urologie oder eine Geburtenhilfe? Wir liefern eine harte Datengrundlage, gegen die man nur schwer argumentieren kann. Wir treffen auch Aussagen zu Investitionen, etwa: Wer hat für wie viel Geld gebaut, was kam am Ende dabei raus, und werden diese Kapazitäten überhaupt gebraucht? Wir liefern erstmals vergleichende Zahlen aus dem primären, sekundären und tertiären Bereich. Der Bericht zeigt, wo Überkapazitäten bestehen. Auf dieser Basis lässt sich die Diskussion über den Abbau von Kapazitäten konkret führen.
Ausgewählte klinische Leistungsgruppen nach Fallzahlen 2011

\begin{tabular}{|c|c|c|c|c|c|c|c|}
\hline \multirow{2}{*}{$\begin{array}{l}\text { Klinische } \\
\text { Leistungsgruppen }\end{array}$} & \multicolumn{7}{|c|}{ Fallzahlen } \\
\hline & KH 1 & KH 2 & KH 3 & KH 4 & KH 5 & KH 6 & KH 7 \\
\hline $\begin{array}{l}\text { Neurologie-Apoplexie } \\
\text { (Schlaganfallbehandlung) }\end{array}$ & 627 & 685 & 328 & 901 & 193 & 869 & 1.302 \\
\hline Lunge/ Thorax-Chirurgisch & 125 & 112 & 20 & 244 & 54 & 49 & 65 \\
\hline Kardiologie/ Elektrophysiologie & 213 & 0 & 16 & & 0 & 65 & 0 \\
\hline Ortho/ Unfall-Wirbelsäule & 1.209 & 957 & 1.054 & 842 & 373 & 1.085 & 619 \\
\hline Leukämie & 76 & 48 & 2 & 30 & 8 & 9 & 57 \\
\hline Polytrauma & 20 & 55 & 9 & 62 & 17 & 34 & 21 \\
\hline
\end{tabular}

Nicht ökonomisch: Der hessische Landesrechnungshof hat die kommunalen Kliniken von Darmstadt, Fulda, Frankfurt, Kassel, Offenbach, des Hochtaunuskreises und des Main-Taunus-Kreises verglichen. Diese Tabelle zeigt, dass einige Klinken Einrichtungen betreiben, in denen nur sehr wenige Patienten behandelt werden. Der Rechnungshof stellt Wirtschaftlichkeit und Qualität dieser Behandlungen in Frage.
Der Bericht kommt zum Schluss, dass vor allem die fünf Häuser im RheinMain-Gebiet kooperieren müssen. Was muss konkret geschehen, und welche Effekte würden sich für die Region ergeben?

In den letzten Jahre haben sich die Krankenhäuser im Ballungsgebiet einen kannibalistischen Wettkampf um Patienten und Leistungsstrukturen geliefert. Unabgestimmt wurden Ressourcen erweitert und neu aufgebaut, ohne dass ein Konzept zur Versorgung der Bevölkerung unter Einbezug auch der anderen Krankenhäuser im Ballungsgebiet, wie die Universitätsklinik oder die der Berufsgenossenschaft, erstellt wurde. Das Resultat ist eine unstrukturierte Versorgungslandschaft mit teuren Doppelvorhaltungen, die aufgrund der geringen Anzahl von Patienten, die in den einzelnen Häusern behandelt werden, vermutlich mit einer schlechteren Qualität einhergeht. Hier muss dringend das Leistungsspektrum abgestimmt und unter qualitativen Gesichtspunkten der Patientenbehandlung reorganisiert werden. Eine solche Strukturveränderung im primären Bereich der Leistungserbringung hätte auch positive ökonomische Aspekte, da unnötige Doppelvorhaltungen vermieden werden könnten. Wesentliche Kostenblöcke in den untersuchten Kliniken sind die Sekundär- und Tertiärbereiche. Hier sollte möglichst unmittelbar eine Abstimmung durchgeführt werden, die sekundären und tertiären Bereiche zu bündeln. Der Effekt wäre eine sofortige wirtschaftliche Gesundung der Häuser im Ballungsgebiet.

Interview: Jens Mau 


\section{Allein 470 Millionen Euro wurden in drei unmittelbar benachbarte Kliniken des Rhein-Main-Gebiets investiert.}

Beachtung gefunden, weil damit die gesundheitspolitisch gewollte Bildung von regionalen Klinikverbünden deutlich erschwert wurde

\section{Nur lahme Enten in die Stiftung?}

Mit einer Stiftung als Eigentümerin wie sie das hessische Konzept vorsieht - wäre die Übernahme der Werra-Meissner-Kliniken durch die GNH am Kartellamt nicht gescheitert, denn die Übernahme wäre gar nicht erst anmeldepflichtig gewesen, schätzt Köhler. Würde die Klinik-Stiftung rechtlich funktionieren, wäre eine Übernahme der Werra-Meissner-Kliniken durch die GNH vielleicht möglich. GNHChef Sontheimer überzeugt das Argument jedoch nicht. „Damit diese Rechnung aufgeht, müssen sehr viele Dinge gleichzeitig klappen“, bemerkt er. Sontheimer möchte der Stiftung ausdrücklich nicht beitreten. Das schmerzt Minister Grüttner und Thomas Köhler. Denn die GNH vertritt mit dem Klinikum Kassel eines der wenigen großen kommunalen Häuser in Hessen, das schwarze Zahlen schreibt. Wieso sollte ein erfolgreich geführtes Krankenhaus mit hoch defizitären Häusern zusammengehen? Eine befriedigende Antwort fehlt. Köhler: „Der operative Bereich des Verbunds muss so aufgestellt werden, dass er sich selbst finanzieren kann. Eine finanzielle Inanspruchnahme der Träger, wie sie heute die Regel ist, darf es nicht geben. Daher müssen alle teilnehmenden Krankenhäuser die gleichen wirtschaftlichen Mindestbedingungen erfüllen. Der Verbund übernimmt einen angemessenen Teil der Verbindlichkeiten und der nicht durchgeführten Investitionen, was die Träger entlastet.“ Er stellt aber auch klar: „Nach seiner Gründung kann und muss dann der Grundsatz „es gibt nur einen gemeinsamen Geldbeutel“ gelten. Nur wenn der Einfluss der Träger in der Klinikstiftung gegen Null geht, kann sie betriebswirtschaftlich sinnvoll entscheiden. Konkret könnte die Stiftung dann so oder ähnlich funktionieren: Der zentrale Einkauf der Stiftung sitzt in Kassel, die Finanzabrechnung in Frankfurt, und regional stimmen die Stiftungskliniken ihr Leistungsangebot ab. So wie die Privaten. „Nicht mehr die einzelne Stadt und der einzelne Landkreis ist damit den $\mathrm{Zu}$ kunftsrisiken ausgesetzt, sondern der Solidargemeinschaft der öffentlichen Hand obliegt als ,Zweckverband' die Erfüllung des Versorgungsauftrags“, unterstreicht Köhler.

\section{Träger diskutieren miteinander}

Rainer Greunke, Geschäftsführer der hessischen Krankenhausgesellschaft, glaubt nicht, dass sich die HessenStiftung durchsetzt: „Ich denke, in regionalen Strukturen nach dem Vorbild der GNH gelingt der Einstieg leichter." Immerhin sei es positiv, dass derzeit eine Diskussion stattfindet. „Stadtkämmerer und Bürgermeister verschiedener Landkreise sprechen miteinander. Das Problembewusstsein erhöht sich." Man darf also gespannt sein, ob sich in Hessen etwas in Richtung Kooperation oder Stiftung bewegt. Positive Beispiele sind vorhanden: Schließlich hat mit Agaplesion einer der größten kirchlichen Verbünde seinen Stammsitz in Frankfurt. Laut Ministerium haben 13 Kliniken Interesse an der Stiftung gezeigt und lassen derzeit begutachten, wie hoch die finanzielle Entlastung der Träger durch einen öffentlichen Krankenhausverbund wäre. Ergebnisse werden Ende August 2013 vorliegen.

Jens Mau
Das Anti-Schwerkraft-Laufband Arte $\mathrm{S}^{\circledR}$ Erleben Sie die Revolution in Rehabilitation und Training!
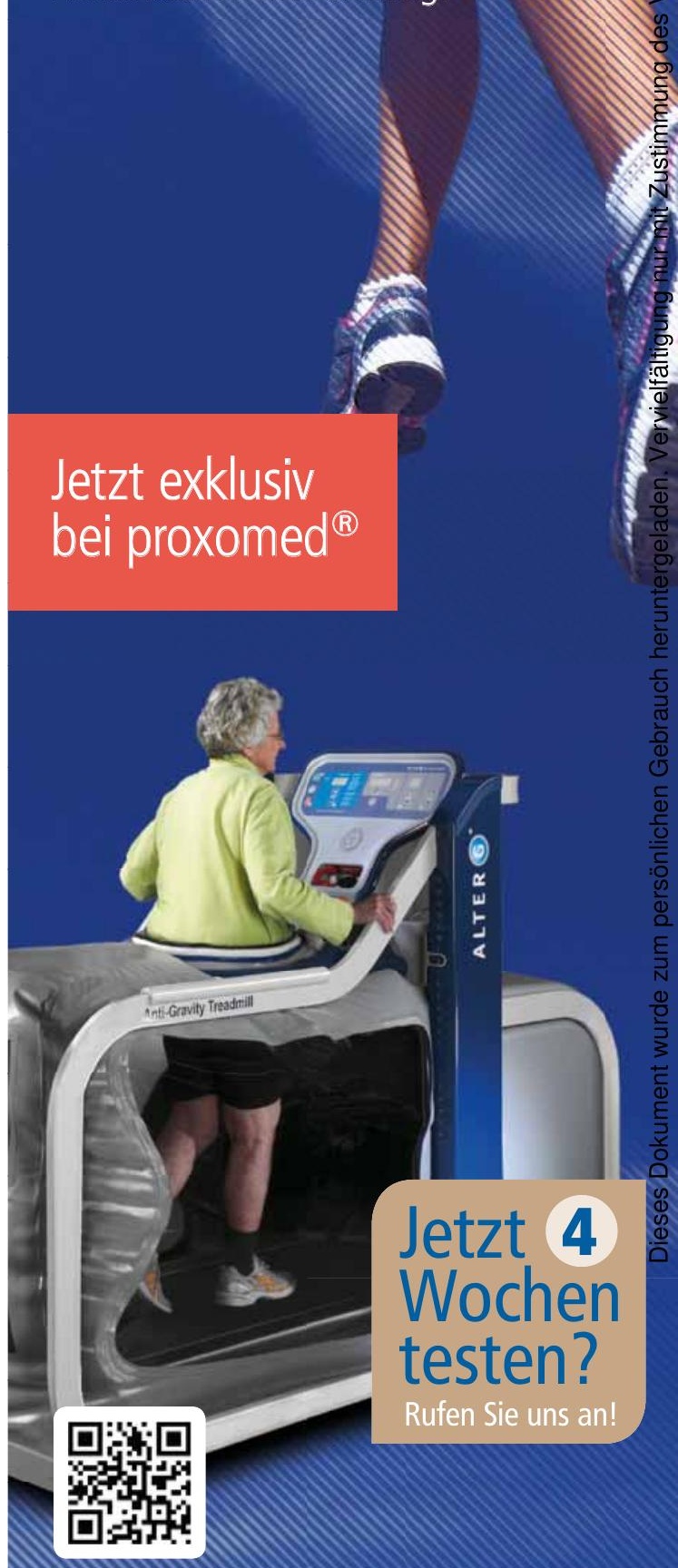

Produktvideo

Telefon: 0602391680

mw. proxomed.com

proxomed Medizintechnik GmbH 